\section{WORLDWIDE PICTURE OF RENAL INVOLVEMENT IN SYSTEMIC LUPUS ERYTHEMATOSUS USING A BIG DATA ANALYTICAL APPROACH}

${ }^{1} \mathrm{M}$ Pérez de Lis Novo, ${ }^{2} \mathrm{M}$ Gandía, ${ }^{3} \mathrm{R}$ Pérez-Álvarez*, ${ }^{4} \mathrm{P}$ Brito-Zerón, ${ }^{5} \mathrm{~B}$ Kostov, ${ }^{6} \mathrm{~A}$ SisoAlmirall, ${ }^{7} \mathrm{D}$ Superville, ${ }^{8} \mathrm{Y}$ Shoenfeld, ${ }^{9} \mathrm{M}$ Ramos-Casals, ${ }^{10} \mathrm{MA}$ Khamashta. ${ }^{1}$ Hospital Juan Canalejo, Anesthesiology and Critical Care, A Coruña, Spain; ${ }^{2}$ Hospital Puerta del Mar, Rheumatology, Cadiz, Spain; ${ }^{3}$ Hospital Alvaro Cunqueiro, Department of Autoimmune Diseases, Vigo, Spain; ${ }^{4}$ Josep Font Laboratory of Autoimmune Diseases- CELLEX-Institut d'Investigacions Biomèdiques August Pi i Sunyer IDIBAPS-, Department of Autoimmune Diseases- ICMiD- Hospital Clinic, Barcelona, Spain; ${ }^{5}$ Primary Care Research Group-Institut d'Investigacions Biomèdiques August Pi i Sunyer IDIBAP, Primary Care Centre Les CortsCAPSE, Barcelona, Spain; ${ }^{6}$ Primary Care Research Group- Institut d'Investigacions Biomèdiques August Pi i Sunyer IDIBAPS, Primary Care Centre Les Corts- CAPSE, Barcelona, Spain: ${ }^{7}$ Massachusetts Institute of Technology, MIT, Cambridge- Massachusetts, USA; ${ }^{8}$ Zabludowicz Centre for Autoimmune Diseases- Chaim Sheba Medical Center- Tel Hashomer-Israel, Incumbent of the Laura Schwarz-Kipp Chair for Research of Autoimmune Diseases- Sackler Faculty of Medicine- Tel-Aviv University, Tel-Aviv, Israel; ${ }^{9}$ osep Font Laboratory of Autoimmune Diseases- CELLEX-Institut d'Investigacions Biomèdiques August Pi i Sunver IDIBAPS-, Department of Autoimmune Diseases- ICMiD- Hospital Clinic, Barcelona, Spain; ${ }^{10}$ St Thomas' Hospital- King's College University, Lupus Research UnitThe Rayne Institute, London, UK

\subsection{6/lupus-2017-000215.417}

Background and aims Lupus nephritis is the most severe involvements of internal organs that may develop patients with systemic lupus erythematosus (SLE). Studying the worldwide distribution of this feature across geographic regions and ethnic groups using a big data-driven approach may help obtain a more high-definition resolution of lupus nephritis

Methods We made a text-word search in Google between 8th and 15th May 2015 using SLE and 100...100000000 patients. We selected the cohorts in which the frequency of renal involvement was detailed, and we analysed the influence on this frequency of the characteristics of the cohorts.

Results Of the 63263 patients included, renal involvement was reported in 24790 patients(38\%). The rate of renal involvement was influenced by the predominant ethnicity:it was lower in cohorts in white patients in comparison with a multiethnic origin $(\mathrm{p}=0.008)$. The mean age at SLE diagnosis was higher in cohorts in which the mean age was $<40$ years $(p=0.019)$. The rate of renal involvement was higher in cohorts with a frequency of positive antiDNA antibodies higher than $75 \%(\mathrm{p}=0.031)$, cohorts with a frequency of positive antiSm antibodies higher than $25 \%(\mathrm{p}=0.046)$, cohorts with a frequency of positive antiRNP antibodies higher than $25 \%(\mathrm{p}=0.031)$ and cohorts with a frequency of positive anti$\mathrm{La} / \mathrm{SSB}$ antibodies higher than $15 \%(\mathrm{p}=0.025)$.

Conclusions This big data analytical approach confirm that the rate of renal involvement is influenced by ethnicity, the mean age at SLE diagnosis and the frequency of positive autoantibodies. The higher rates of renal involvement being found in US and Asian studies, with the youngest patients at the time of SLE diagnosis and highest rates of immunopositive patients.

\section{PRINCIPAL COMPONENT ANALYSIS AS A METHOD TO UNRAVEL THE RELATION BETWEEN DISEASE ACTIVITY AND CYTOKINE PROFILING IN SYSTEMIC LUPUS ERYTHEMATOSUS}

${ }^{1} \mathrm{~W}$ Raymond*, ${ }^{2} \mathrm{G}$ Eilertsen, ${ }^{1} \mathrm{~J}$ Nossent. ${ }^{1}$ The University of Western Australia, Rheumatology Group - School of Medicine, Perth, Australia; ${ }^{2}$ Arctic University of Tromso, Rheumatology, Tromso, Norway

10.1136/lupus-2017-000215.418

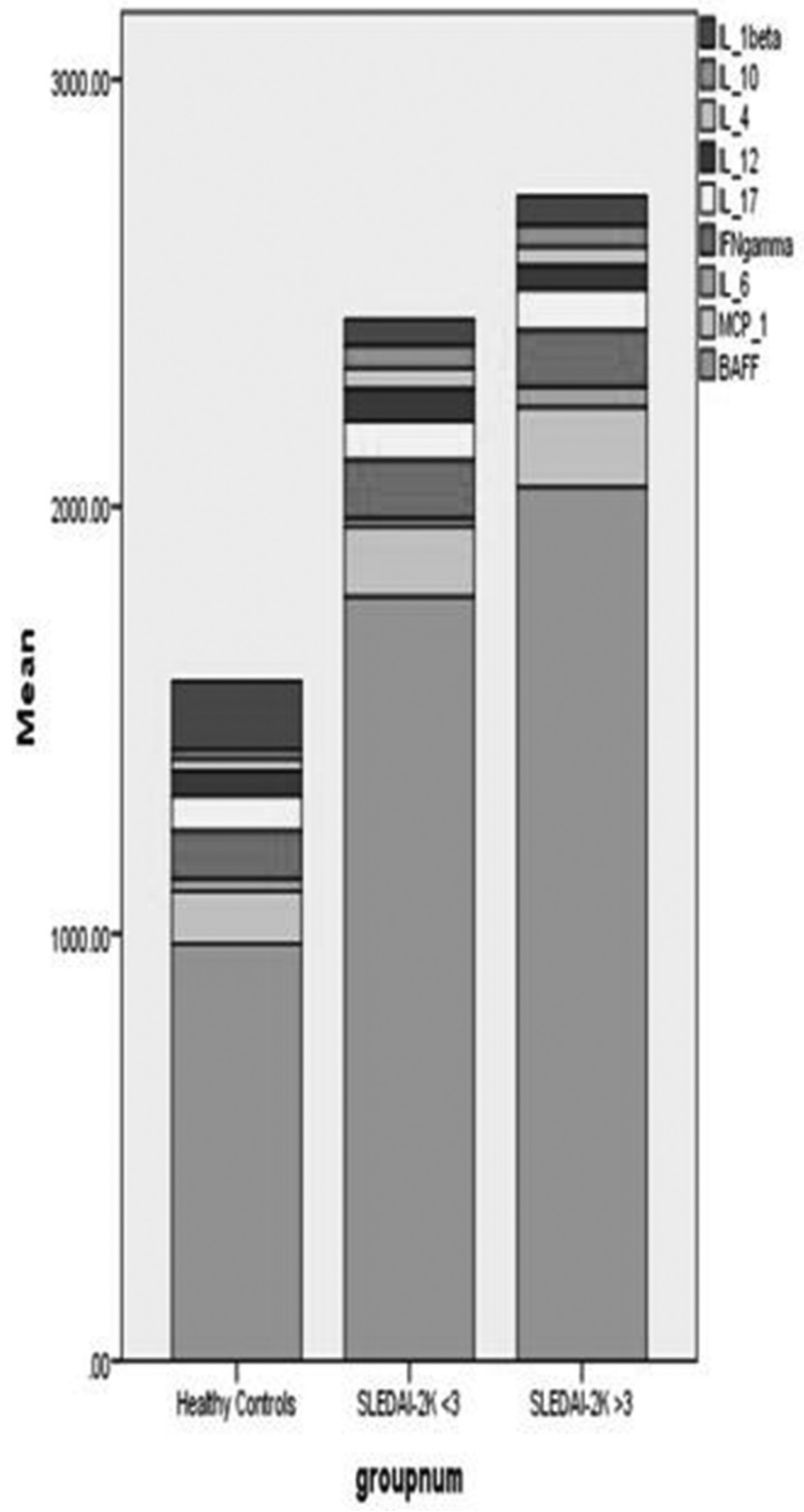

Abstract 418 Figure 1 Mean serum levels $(\mathrm{pg} / \mathrm{mL})$ of cytokines in heathly controls and SLE patients.

Background Cytokine dysregulation contributes to immune dysfunction, inflammation and organ damage in Systemic Lupus Erythematosus (SLE). Cytokines have multifarious interactions on other cytokines and immune cells. Thus cytokines studies should reflect this intrinsic complexity.

Aim Utilise Principle Component Analysis (PCA) to unravel the interplay of a selection of cytokines for SLE versus healthy controls.

Methods A cross-sectional study of 102 SLE patients and 30 controls. Sera samples of IFN- $\gamma$, IL-1 $\beta$, IL-4, IL-6, IL-10, IL12, IL17, BAFF and MCP-1, were analysed by ELISA and compared non-parametrically between groups. SLE disease activity was assessed by SLEDAI-2K. PCA demonstrated the cytokine profiles of healthy controls, SLE patients with low $($ SLEDAI- $2 \mathrm{~K}<3)$ and moderate to high disease activity (SLEDAI- $2 \mathrm{~K} \geq 3$ ).

Results BAFF correlated with disease activity (Rs. 0.483, $\mathrm{p}<0.001)$ and highest in SLEDAI- $2 \mathrm{~K} \geq 3(\mathrm{p}<0.001)$. BAFF was 
Abstract 418 Table 1 Median serum levels of cytokines $(\mathrm{pg} / \mathrm{mL}$ ) in Controls and SLE patients with SLEDAI-2K $<3$ and SLEDAI-2K $\geq 3$. IQR; Interquartile range.

\begin{tabular}{|c|c|c|c|c|}
\hline & \multicolumn{3}{|c|}{ Cohort } & \multirow{2}{*}{$\begin{array}{c}\text { Independent- } \\
\text { Samples } \\
\text { Kruskal-Walis } \\
\text { test }\end{array}$} \\
\hline & Healthy Controls & SLEDAI-ZK $\& 3$ & SLEDAI-2K $>3$ & \\
\hline Cytokine & Median (IQR) & Median (IQR) & Median (IQR) & p-value \\
\hline BAFF & $980(750,1150)$ & $1590(1210,2230)$ & $1170(1320,2470)$ & $\$ 0.001$ \\
\hline IFN- & $47.3(19.6,113.9)$ & $75.4(19.6,140.1)$ & $57.8(19.6,128.0)$ & 0.759 \\
\hline$\| \cdot 1 B$ & $17.9(17.9,269.0)$ & $17.9(17.9,17.9)$ & $17.9(17.9,17.9)$ & 0.001 \\
\hline 11.4 & $7(7,7)$ & $7(7,7)$ & $7(7,7)$ & 0.858 \\
\hline 11.6 & $14(14,15.7)$ & $14(14,14)$ & $14(14,21.9)$ & 0.448 \\
\hline$\| \cdot-10$ & $5.9(5.9,17.6)$ & $5.9(5.9,11.4)$ & $5.9(5.9,23.3)$ & 0.68 \\
\hline $16 \cdot 12$ & $40.4(12.6,86.2)$ & $34.2(12.6,66.0)]$ & $14.7(12.6,60.5)$ & 0.162 \\
\hline MCP-1 & $102.4(45.3,195.7)$ & $145.1(84.0,218.6)$ & $131.0(75.7,221.9)$ & 0.179 \\
\hline$\|:-17$ & $28.4(28.4,94.9)$ & $28.4(28.4,42)$ & $28.4(28.4,69.4)$ & 0.929 \\
\hline
\end{tabular}

not in the Principal Component 1 (PC1) of any group $(-0.22$ vs -0.18 vs 0.06$)$ (Figures 2 and 3 ).

IL-1 $\beta$ was inversely correlated with disease activity (Rs. $-0.216, p=0.013)$ and lowest in SLEDAI- $2 \mathrm{~K} \geq 3 \quad(\mathrm{p}=0.001)$. IL-1 $\beta$ was a moderate driver of cytokine variance for healthy controls, but became more dominant across SLEDAI- $2 \mathrm{~K}<3$ and SLEDAI-2K $\geq 3$, i.e. PC1 ( 0.59 vs 0.976 vs 0.985 ).

Conclusions Increased BAFF levels were not a direct agitator of cytokine variation in SLE, suggesting a contribution to disease activity through other pathways. In contrast, the reduction of IL-1 $\beta$ had a dominant effect on cytokine variance in SLE (PC 1). Principal component analysis is a useful asset for cytokine profiling in SLE.

\section{HOSPITALISATION FOR SYSTEMIC LUPUS ERYTHEMATOSUS IN WESTERN AUSTRALIA DOUBLES THE ALL-CAUSE MORTALITY RISK COMPARED TO CONTROLS, ESPECIALLY FOR MEDICARE RELIANT AND MALE PATIENTS}

${ }^{1}$ W Raymond*, ${ }^{2} \mathrm{D}$ Preen, ${ }^{1} \mathrm{H}$ Keen, ${ }^{3} \mathrm{C}$ Inderjeeth, ${ }^{1} \mathrm{~J}$ Nossent. ${ }^{1}$ The University of Western Australia, Rheumatology Group - School of Medicine, Perth, Australia; ${ }^{2}$ The University of Western Australia, School of Population Health, Perth, Australia; ${ }^{3}$ Sir Charles Gairdner and Osborne Park Hospital Group, Rheumatology, Perth, Australia
Background and aims Hospitalisation for Systemic Lupus Erythematosus (SLE) is a significant event. We aimed to understand the factors leading to an incident admission and its impact on long term outcome in SLE patients in Western Australia (WA).

Methods Using whole-population data linkage of hospital admissions, cancer registrations and death records in WA from 1980 to 2015, we performed a retrospective comparative analysis for all patients admitted with a first ever primary diagnosis of SLE (ICD-9-CM 695.4, 710.0, ICD-10-AM L93.0 and M32). For SLE patients, we analysed annual incident hospitalisation rates and compared patient characteristics, all-cause mortality and cancer risk (by Kaplan-Meier and Cox regression) versus age- and gender-matched controls free of rheumatic disease.

Results The incident hospitalisation rate for SLE (mean 20.9/ million/year (CI: 11-35) showed little variation. SLE patients were younger, more likely to be Indigenous, uninsured, have kidney and cardiovascular disorders and to die during their first hospitalisation (Table 1). Cancer risk was equivalent, but a first admission for SLE doubled the risk of subsequent death $(\mathrm{OR}=1.99$, CI: 1.5-2.7, $\mathrm{p}<0.001)$ (Figure 1, Table 2). Medicare reliance (OR 1.7, CI: 1.4-1.9, $\mathrm{p}=0.001$ ) and male gender (OR 1.4, CI: 1.0-1.8, p<0.04) were the strongest independent predictors of death. 